\title{
Photometric Variability of OB-type stars as a New Window on Massive Stars
}

\author{
M. Kourniotis ${ }^{1,2}$, A. Z. Bonanos ${ }^{1}$, I. Soszyński ${ }^{3}$, \\ R. Poleski ${ }^{3,4}$, G. Krikelis ${ }^{2}$ and the OGLE team \\ ${ }^{1}$ IAASARS, National Observatory of Athens, GR-15236 Penteli, Greece \\ email: mkourniotis@astro.noa.gr, bonanos@astro.noa.gr \\ ${ }^{2}$ Section of Astrophysics, Astronomy and Mechanics, Faculty of Physics, University of Athens, \\ Panepistimiopolis, GR15784 Zografos, Athens, Greece \\ ${ }^{3}$ Warsaw University Observatory, Al. Ujazdowskie 4, 00-478 Warszawa, Poland \\ ${ }^{4}$ Department of Astronomy, Ohio State University, 140 W. 18th Ave., Columbus, OH 43210, \\ USA
}

\begin{abstract}
We present the first systematic study of 4646 spectroscopically confirmed early-type massive stars in the Small Magellanic Cloud (SMC), using variability as a tool to confine the physics of OB-type massive stars. We report the discovery of $\sim 100$ massive eclipsing systems which are useful for the accurate determination of the fundamental parameters of massive stars and we evaluate the frequency of multiplicity. In addition, we explore the occurrence of the $\mathrm{Oe} / \mathrm{Be}$ phenomenon and provide a large number of candidate non-radial pulsators, which can be further studied via asteroseismology. The results of this work (Kourniotis et al. 2014) will contribute to a better understanding of the role of metallicity in triggering processes associated to matter ejections and/or disk formation, which in turn affect mass loss and stellar rotation.
\end{abstract}

Keywords. stars: emission-line, Be, (stars:) binaries: eclipsing, galaxies: individual (SMC), stars: variables: other

\section{Introduction}

Photometric variability has been acclaimed to be a unique tool to constrain the physics of hot massive stars. Extended photometric surveys have already yielded large catalogs of eclipsing binaries (EBs) and pulsating variables in the Galaxy and nearby galaxies. Eruptive events due to strong photometric processes have been monitored, allowing a deep insight on the nature of massive variables such as Be stars. The occurrence and intensity of the Be phenomenon has been already reported to be higher in low metallicity environments owing to the lower mass loss rates that result in lower angular momentum losses (Vink et al. 2001). Consequently, massive stars reach a high $\Omega / \Omega_{c}$ ratio, triggering the formation of an equatorial disk, the modification of which results in photometric and spectroscopic variability (Rivinius et al. 2013).

The low metallicity of the SMC provides an ideal laboratory in the context of evaluating the frequency of Be stars among environments of different metallicity. Motivated by the availability of a large photometric database with long-term lightcurves provided by the wide-field monitoring of the OGLE-III survey, as well as of an extended catalog of 5324 massive stars with known spectral types in the SMC (Bonanos et al. 2010), we aim to exploit the time domain in order to identify photometrically candidate Be stars, report newly discovered eclipsing binaries and hot, candidate pulsators and correlate variability with the spectral type of massive stars in the SMC. 


\section{Input Catalogues and Analysis}

The input catalog of 5324 massive stars in the SMC provided by Bonanos et al. (2010), consists of 12 Wolf Rayet, 3983 OB-type stars and 1329 stars of later spectral types, with a typical accuracy to one luminosity class and one spectral sub-type. The OGLE-III observations were conducted between 2001 and 2009 with the 1.3-m Warsaw telescope at Las Campanas Observatory in Chile that covered 14 square degrees in the direction of the SMC (Udalski et al. 2008). High-quality light curves were provided in the Cousins $I$-band and Johnson $V$ photometric band and matched with the stars of the input catalogue. Very bright and thus saturated stars as well as stars that were located out of the OGLE-III field were excluded, resulting in a final list of 4646 stars with $I$-band magnitudes between 12.6 - 19.4 mag.

As a first step, the light curves were studied with respect to their mean magnitude and standard deviation. We used the $\sigma_{I}$ vs. $I$ plane and imposed an empirical curve based on the OGLE accuracy, following the method proposed by Graczyk \& Eyer (2010) to distinguish between real variables and non-variables (Fig. 1). A second, shifted curve was added to the plane to further confine stars with high-amplitude fluctuations mainly attributed to the Be mechanism. We then flagged as high-amplitude variables those stars located above our suggested curve, low-amplitude those found between the two curves and "constant" stars those that lie below the primary, empirical curve. Eclipsing binaries were identified following the method introduced by Graczyk \& Eyer (2010) based on the skewness and the kurtosis of the light curves. We focused on the region of the skewness-kurtosis plane where we expect EBs to lie and searched the light curves for periodic signals using the Analysis of Variance (AoV) algorithm (Schwarzenberg-Czerny 1989). We then folded the light curves to the period that corresponds to the frequency with the highest peak in the power spectrum, yielding $\sim 200$ EBs and a large number of sinusoidally varying periodic stars representative of pulsators and rotational variables.

\section{Results}

Following the previously described periodic search, we identified 205 eclipsing binaries, 101 of which are newly reported for their eclipsing nature. We assign the spectral type to 59 known EBs and derive periods, which in many cases are more precise than those reported in the literature owing to their long-term monitoring. Among the identified EBs, we further report rare cases of transient EBs, double-periodic variables considered to be systems surrounded by a circumbinary disk and binaries with evident indication of apsidal motion.

A total of 249 periodic stars present sinusoidal behaviour with equal minima, corresponding to pulsators and rotating variables. We classified these stars as "short" and "long period" variables, when displaying periods shorter and longer than 3 days, respectively. We then flagged variables as "periodic with extra variability" when their periodic signal varies in amplitude or baseline by more than 0.05 mag (see Fig. 8, Kourniotis et al. 2014). The remaining periodic variables consist of 126 classical Cepheids and 50 stars flagged as "rotating variables" being sinusoidally periodic variables with unequal minima which implies a strong indication of tidal distortion effects for an ellipsoidal star or starspot modulation. 


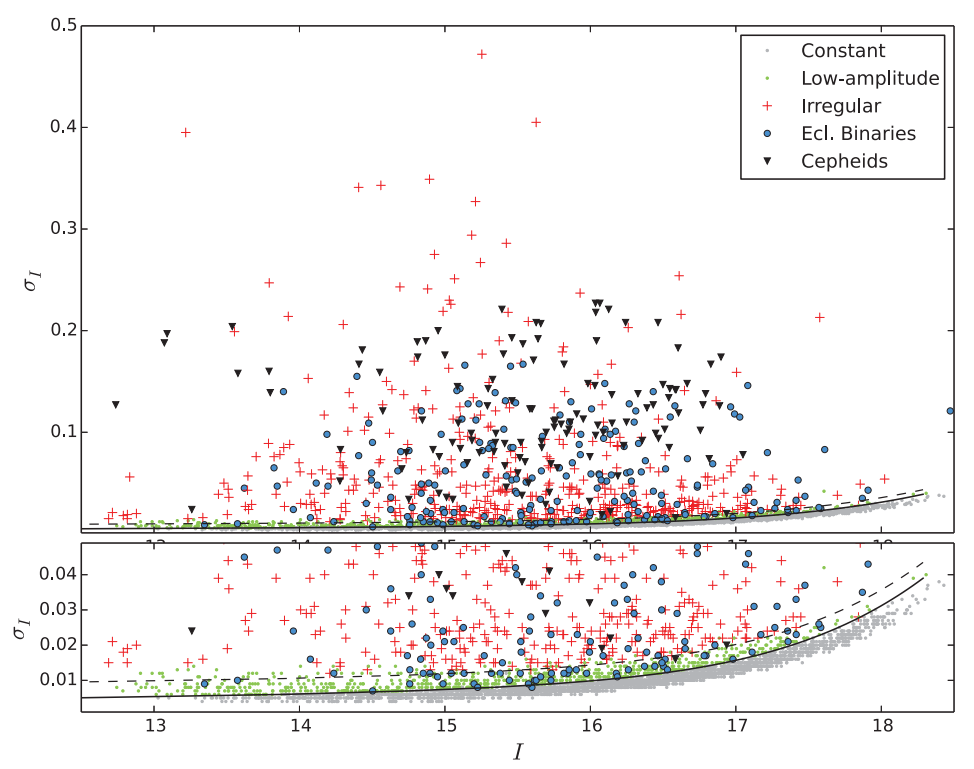

Figure 1. $\sigma_{I}$ vs. $I$ diagram for our studied stars. The solid curve is proposed to differentiate real from spurious variables (Graczyk \& Eyer 2010). Low-amplitude variables are located between the proposed and our suggested (dashed) curve. A zoomed region is presented in the lower panel.

Our high-amplitude, stochastic variables flagged as "irregular", on their majority display a combination of short or long-term outbursts and/or trends. We distinguished 4 modes of variability similar to the modes presented in Keller et al. (2002) for classifying the blue variables in the LMC, that consist of high-amplitude, long-term outbursts ("bumper" events), short-term and low-amplitude, sudden outbursts ("flicker" events), monotonic trends across the 8-year time domain ("monotonic") and decline of magnitude that lasts up to 1000 days ("fading" events) (see Fig. 9, Kourniotis et al. 2014). Of our 443 irregular variables, $\sim 76 \%$ are newly reported as variables in the present work.

\section{Discussion}

We proceed to study the variability of 4646 stars as defined in the previous section, with respect to the spectral type. Fig. 2 presents 4 pie charts showing the distribution of variability for $\mathrm{O}$, early $\mathrm{B}$, late $\mathrm{B}$ and AFG-type stars. The most prominent feature is the decline of variability -particularly of "irregular" type- beyond the B2 spectral type. Short-term periodic stars are concentrated in early-type spectral bins whereas eclipsing binaries are mostly frequent in O-type stars, in agreement with the high binarity frequency reported among stars of this type (Sana et al. 2012).

The majority of the irregular variables exhibit reddening, as shown in Fig. 3, which presents a color-magnitude diagram (CMD) for our studied stars with respect to the type of variability, whereas low-amplitude variability in O-type stars is mainly restricted to the blue regime possibly owing to wind variability. We propose irregular and low-amplitude variability for B-type stars to be a photometric criterion for defining a candidate Be star. Applying this criterion to our stars, the fraction of candidate early Be stars is found to be $30 \pm 1 \%$, consistent with the respective fractions derived in the literature using different methods. 

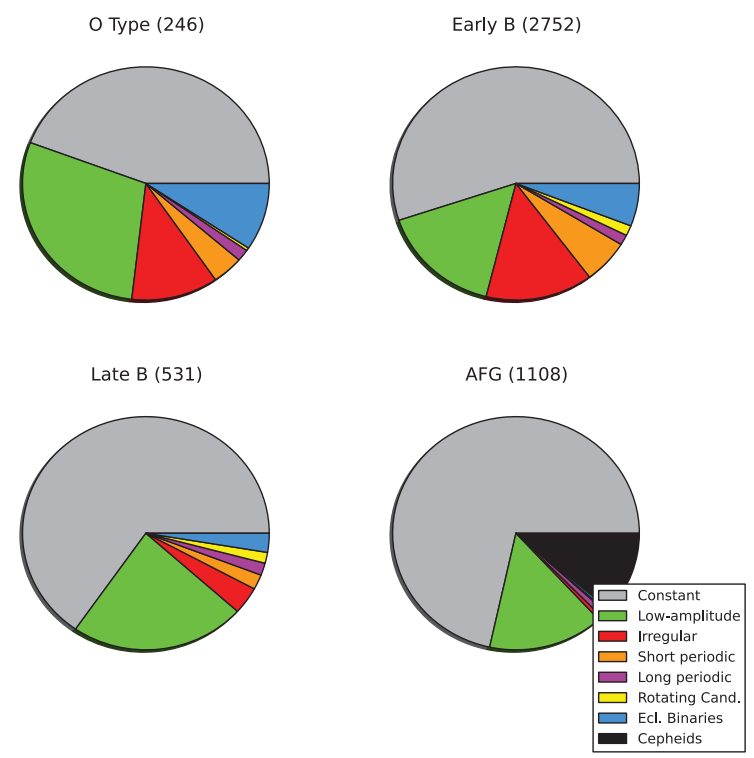

Figure 2. Pie charts displaying the distribution of variability for our studied O, early B, late B and AFG-type stars.

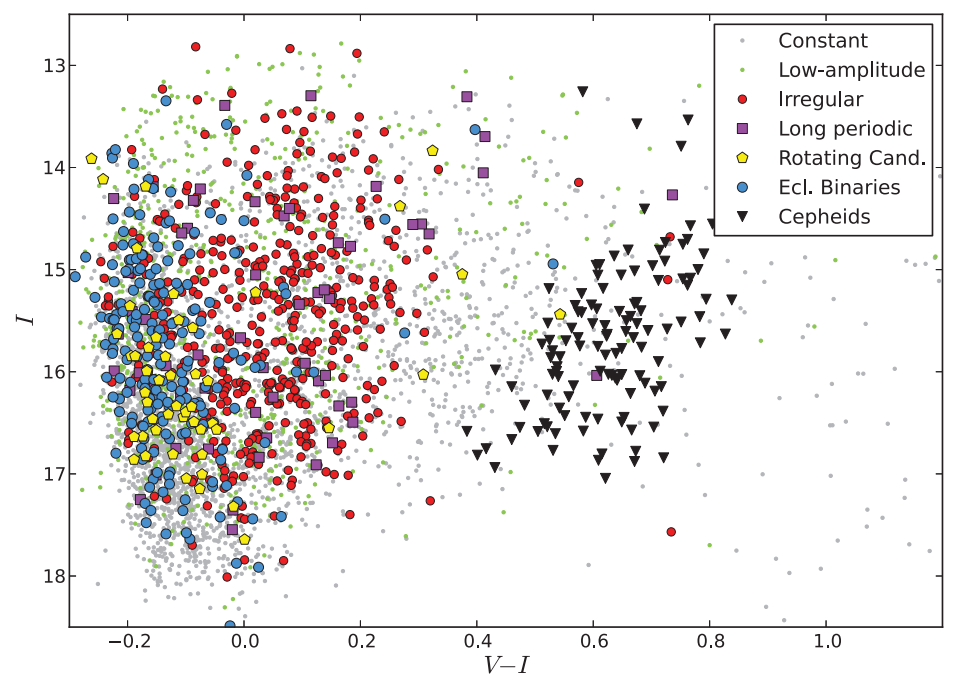

Figure 3. Color-magnitude diagram of our studied stars for the type of variability.

Of our spectroscopically confirmed Be stars, $78 \pm 4 \%$ exhibit prominent variability on the 8-year time domain of OGLE-III i.e. irregular, low-amplitude or short-term periodic variability. We caution that since the fraction of short-term periodic variables is incomplete and that low-amplitude variables may have a smaller chance of being detected with $\mathrm{H} \alpha$ emission during spectrscopy than irregular variables, the derived fraction of photometrically variable Be stars corresponds to a lower limit.

Early-type short-term periodic variables of mainly low-amplitude, occupy distinctive regions in the CMD where the known Slowly Pulsating B stars (SPBs) and non-radially pulsating Be variables of the SMC are located (see Fig. 17, Kourniotis et al. 2014). They 
thus provide ground for further examination of the properties of the metal opacity bump in low metallicity environments (Salmon et al. 2012). Having examined 4 pulsating Be stars with extra variability as defined in Sec. 3, we find that they are rotating close to their critical speed, likely enhancing matter ejections that cause the observed additional variability.

\section{References}

Bonanos, A. Z., Lennon, D. J., Köhlinger, F., et al. 2010, AJ 140, 416

Graczyk, D. \& Eyer, L. 2010, AcA 60, 109

Keller, S. C., Bessell, M. S., Cook, K. H., Geha, M., \& Syphers, D. 2002, AJ 124, 2039

Kourniotis, M., Bonanos, A. Z., Soszyński, I., et al. 2014, A\&\&A 562, A125

Rivinius, T., Carciofi, A. C., \& Martayan, C. 2013, A\&A Rev. 21, 69

Salmon, S., Montalbán, J., Morel, T., et al. 2012, MNRAS 422, 3460

Sana, H., de Mink, S. E., de Koter, A., et al. 2012, Science 337, 444

Schwarzenberg-Czerny, A. 1989, MNRAS 241, 153

Udalski, A., Soszyński, I., Szymański, M. K., et al. 2008, AcA 58, 329

Vink, J. S., de Koter, A., \& Lamers, H. J. G. L. M. 2001, A\&\&A 369, 574

\section{Discussion}

Fossati: The fraction of rotating variables among O-type stars appears to be quite small. Could that be due to selection effects?

Kourniotis: We denoted as candidate rotating variables those sinusoidally periodic stars with unequal minima as this implies indication of starspot modulation. The fraction of candidate rotating variables is incomplete, since "constant" stars were not searched for periodic signals and hence, we discard from our study rotating variables with very low amplitude. Rotating variables shall be also found among our short/long periodic variables. Therefore, the particular fraction shall not be considered as intrinsic but rather, as a lower limit. 